\title{
To find a non-split strong dominating set of an interval graph using an algorithm
}

\author{
Dr. A. Sudhakaraiah*, A. Sreenivasulu ${ }^{1}$, V. Rama Latha ${ }^{2}$, E. Gnana Deepika ${ }^{3}$, \\ Department of Mathematics, S. V. University, Tirupati-517502, Andhra Pradesh, India.
}

\begin{abstract}
In graph theory, a connected component of an undirected graph is a sub graph in which any two vertices are connected to each other by paths. For a graph $G$, if the subgraph of $G$ itself is a connected component then the graph is called connected, else the graph $G$ is called disconnected and each connected component sub graph is called it's components. A dominating set $D_{\text {st }}$ of graph $G=(V, E)$ is a non-split strong dominating set if the induced sub graph $\left\langle V-D_{s t}\right\rangle$ is connected. The non-split strong domination number of $G$ is the minimum cardinality of a non-split strong dominating set. In this paper constructed a verification method algorithm for finding a non-split strong dominating set of an interval graph.
\end{abstract}

Keywords: Domination number, Interval graph, Strong dominating set, Strong domination number, split dominating set.

\section{Introduction}

Let $\mathrm{I}=\left\{\mathrm{I}_{1}, \mathrm{I}_{2}, \ldots ., \mathrm{I}_{\mathrm{n}}\right\}$ be the given interval family. Each interval $\mathrm{i}$ in $\mathrm{I}$ is represented by $\left[a_{i}, b_{i}\right]$, for $i=1,2, \ldots ., n$. Here $a_{i}$ is called the left endpoint and $b_{i}$ the right endpoint of the interval $\mathrm{I}_{\mathrm{i}}$.Without loss of generality we may assume that all end points of the intervals in $I$ which are distinct between 1 and $2 n$. The intervals are labelled in the increasing order of their right endpoints. Two intervals $i$ and $j$ are said to intersect each other, if they have non-empty intersection. Interval graphs play important role in numerous applications, many of which are scheduling problems. A graph $G=(V, E)$ is called an interval graph if there is a one-to-one correspondence between $V$ and I such that two vertices of $G$ are joined by an edge in $E$ if and only if their corresponding intervals in I intersect. That is, if

$i=\left[a_{i}, b_{i}\right]$ and $j=\left[a_{j}, b_{j}\right]$, then $i$ and $\mathrm{j}$ intersect means either $a_{j}<b_{i}$ or $a_{i}<b_{j}$.

Let $G$ be a graph, with vertex set $V$ and edge set $E$.

The open neighbourhood set of a vertex $v \in V$ is $n b d(v)=\{u \in V / u v \in E\}$.

The closed neighbourhood set of a vertex $v \in V$ is $n b d[v]=n b d(v) \cup\{v\}$.

A vertex in a graph $\mathrm{G}$ dominates itself and its neighbors. A set $D \subseteq V$ is called dominating set if every vertex in $\langle V-D\rangle$ is adjacent to some vertex in D. The domination studied in [1-2]. The domination number $\gamma$ of $\mathrm{G}$ is the minimum cardinality of a dominating set. The domination number is well-studied parameter. We can see this from the bibliography [3] on domination. In [4] , Sampathkumar and Pushpa Latha have introduced the concept of strong domination in graphs. Strong domination has been studied [5-7]. Kulli. V. R. et all [8] introduced the concept of split and non-split domination[9] in graphs. Also Dr.A. Sudhakaraiah et all [10] discussed an algorithm for finding a strong dominating set of an interval graph using an algorithm . A dominating set $D$ is called split dominating set if the induced subgraph $\langle V-D\rangle$ is disconnected. The split domination number of $\gamma_{s}$ of $G$ is the minimum cardinality of a split dominating set. Let $G=(V, E)$ be a graph and $u, v \in V$.

Then u strongly dominates $v$ if

(i) $u v \in E$

(ii) $\operatorname{deg} v \leq \operatorname{deg} u$.

A set $\mathrm{D}_{\mathrm{st}} \subseteq \mathrm{V}$ is a strong dominating set of $G$ if every vertex in $V-D_{s t}$ is strongly dominated by at least one vertex in $D_{s t}$. The strong domination number $\gamma_{s t}(G)$ of $G$ is the minimum cardinality of a strong dominating set. A dominating set $D_{s t} \subseteq V$ of a graph $G$ is a Non-split strong dominating set if the induced subgraph $<V-D>$ is connected.Define $N I(i)=j$, if $\mathrm{b}_{i}<a_{j}$ and there do not exist an interval $k$ such that $b_{i}<a_{k}<a_{j}$. If there is no such $j$, then define $N I(i)=$ null. $\mathrm{N}_{\mathrm{sd}}(\mathrm{i})$ is the set of all neighbors whose degree is greater than degree 
of $i$ and also greater than i.If there is no such neighbor then defines $N_{s d}(i)=n u l l . \mathrm{M}(\mathrm{S})$ is the largest highest degree vertex in the set $\mathrm{S}$.

\section{Algorithms.}

2.1.To find a Strong dominating set (SDS) of an interval graph using an algorithm[9]. Input : Interval family $I=\left\{I_{1}, I_{2}, \ldots \ldots, I_{n}\right\}$.

Output : Strong dominating set of an interval graph of a given interval family.

Step $1: S_{1}=n b d[1]$.

Step $2: \mathrm{S}=$ The set of vertices in $\mathrm{S}_{1}$ which are adjacent to all other vertices in $\mathrm{S}_{1}$.

Step $3: \mathrm{D}_{\text {st }}=$ The largest highest degree interval in $\mathrm{S}$.

Step $4: \mathrm{LI}=$ The largest interval in $\mathrm{D}_{s t}$

Step 5 : If $\mathrm{N}_{s d}(L I)$ exists

Step $5.1: \mathrm{a}=\mathrm{M}\left(\mathrm{N}_{s d}(L I)\right)$.

Step $5.2: \mathrm{b}=$ The largest highest degree interval in nbd [a].

end if

Step $5.3: \mathrm{D}_{s t}=D_{s t} \cup\{b\}$ goto step 4 .

else

Step 6 : Find NI(LI)

Step 6.1: If NI(LI) null goto step 7 .

Step $6.2: \mathrm{S}_{2}=n b d[N I(L I)]$.

Step $6.3: \mathrm{S}_{3}=$ The set of all neighbors of $N I(L I)$ which are greater than or equal to $N I(L I)$.

Step $6.4: \mathrm{S}_{4}=$ The set of all vertices in $\mathrm{S}_{3}$ which are adjacent to all vertices in $\mathrm{S}_{3}$.

Step $6.5: \mathrm{c}=$ The largest highest degree interval in $\mathrm{S}_{4}$.

Step $6.6: \mathrm{D}_{s t}=D_{s t} \cup\{c\}$ goto step 4 .

Step 7 : End.

2.2.To find a Non-split Strong dominating set (NSSDS) of an interval graph using an algorithm. Input : Interval family $\mathrm{I}=\left\{\mathrm{I}_{1}, \mathrm{I}_{2}, \mathrm{I}_{3},---------\mathrm{I}_{\mathrm{n}}\right\}$.

Output : Whether a strong dominating set is a non split strong dominating set or not.

Step1 : $\mathrm{S}_{1}=\mathrm{nbd}[1]$

Step2 : $S=$ The set of vertices in $S_{1}$ which are adjacent to all other vertices in $S_{1}$.

Step3 : $\mathrm{D}_{\mathrm{st}}=$ The largest highest degree interval in $\mathrm{S}$.

Step4 : LI=The largest interval in $\mathrm{D}_{\mathrm{st}}$

Step5 : If $\mathrm{W}_{\mathrm{sd}}(\mathrm{LI})$ exists

Step $5.1: \mathrm{a}=\mathrm{M}\left(\mathrm{N}_{\mathrm{sd}}(\mathrm{LI})\right)$

Step 5.2: $\mathrm{b}=$ The largest highest degree interval in nbd[a]

End if

Step $5.3: D_{\text {st }}=D_{\text {st }} \cup\{b\}$ go to step 4

Else

Step 6 : Find NI(LI).

Step $6.1:$ If $\mathrm{NI}(\mathrm{LI})=$ null go to step 7 .

Step $6.2: \mathrm{S}_{2}=\mathrm{nbd}[\mathrm{NI}(\mathrm{LI})]$

Step $6.3: \mathrm{S}_{3}=$ The set of all neighbors of $N I(L I)$ which are greater than or equal to $N I(L I)$.

Step $6.4: \mathrm{S}_{4}=$ The set of all vertices in $\mathrm{S}_{3}$ which are adjacent to all vertices in $\mathrm{S}_{3}$.

Step $6.5: \mathrm{c}=$ The largest highest degree interval in $\mathrm{S}_{4}$.

Step $6.6: \mathrm{D}_{s t}=D_{s t} \cup\{c\}$ goto step 4 .

Step $7: \mathrm{V}=\{1,2,3,----------, \mathrm{n}\}$

Step $8:\left|D_{\text {st }}\right|=\mathrm{k}$

Step 9: $S_{N}=\left\{V-D_{\text {st }}\right\}=\left\{S_{1}, S_{2}, S_{3},---------, S_{k}\right\}, k_{1} \leq n-k$ 


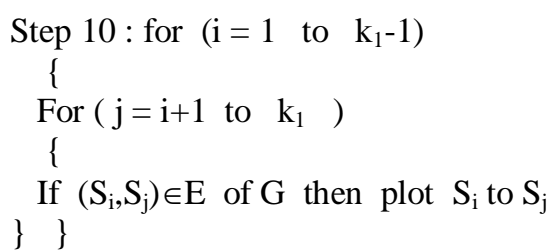

The induced sub graph $\mathrm{G}_{1}=\mathrm{V}-\mathrm{D}_{\mathrm{st}}$ is obtained

Step11 : If $\mathrm{W}\left(\mathrm{G}_{1}\right)=1$

$\mathrm{D}_{\mathrm{st}}$ is non split strong dominating set

Else

$\mathrm{D}_{\mathrm{st}}$ is split strong dominating set

End.

\section{Main Theorems}

Theroem 1 : Let $G$ be an interval graph corresponding to an interval family $I=\left\{I_{1}, I_{2}, I_{3},------I_{n}\right\}$. If $i$ and $j$ are any two intervals in I such that $i \in D_{\text {st }}$ is minimum strong dominating set of the given interval graph $G, j \neq 1$ and $j$ is contained in $i$ and if there is at least one interval to the left of $j$ that intersects $j$ and at least one interval $k \neq i$ to the right of $j$ that intersects $j$ then $D_{s t}$ is a non split strong domination.

Proof : Let $G$ be an interval graph corresponding to an interval family $I=\left\{I_{1}, I_{2}, I_{3},-----I_{n}\right\}$. Let $i$ and $j$ be any two intervals in I such that $i \in D_{\text {st }}$, where $D_{\text {st }}$ is a minimum strong dominating set of the given interval graph $G$, $\mathrm{j} \neq 1$ and $\mathrm{j}$ is contained in $\mathrm{i}$ and suppose there is at least one interval to the left of $\mathrm{j}$ that intersects $\mathrm{j}$ and at least one interval $\mathrm{k} \neq \mathrm{i}$ to the right of $\mathrm{j}$ that intersects $\mathrm{j}$. Then it is obviously we know that $\mathrm{j}$ is adjacent to $\mathrm{k}$ in the induced subgraph $\left\langle\mathrm{V}-\mathrm{D}_{\mathrm{st}}\right\rangle$.Then there will be a connection in $\left\langle\mathrm{V}-\mathrm{D}_{\mathrm{st}}\right\rangle$ to its left.

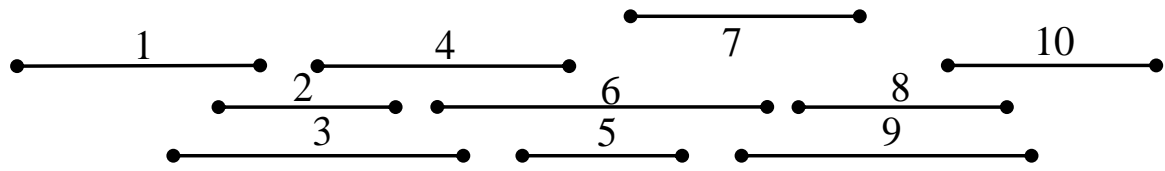

Interval family I

As follows an algorithm with illustration for neighbours as given interval family I. We construct an interval graph $\mathrm{G}$ from interval family $\mathrm{I}=\{1,2,3,-------10\}$ as follows

$\operatorname{nbd}[1]=\{1,2,3\}, \quad \operatorname{nbd}[2]=\{1,2,3,4\}, \quad \operatorname{nbd}[3]=\{1,2,3,4,6\}$,

$\operatorname{nbd}[4]=\{2,3,4,5,6\}, \quad \operatorname{nbd}[5]=\{4,5,6,7\}, \quad \operatorname{nbd}[6]=\{3,4,5,6,7,9\}$,

$\operatorname{nbd}[7]=\{5,6,7,8,9\}, \quad \operatorname{nbd}[8]=\{7,8,9,10\}, \quad \operatorname{nbd}[9]=\{6,7,8,9,10\}$,

$\operatorname{nbd}[10]=\{8,9,10\}$.

$\mathrm{N}_{\mathrm{sd}}(1)=\{2,3\}, \quad \mathrm{N}_{\mathrm{sd}}(2)=\{3,4\}, \mathrm{N}_{\mathrm{sd}}(3)=\{6\}, \quad \mathrm{N}_{\mathrm{sd}}(4)=6, \quad \mathrm{~N}_{\mathrm{sd}}(5)=\{6\}, \quad \mathrm{N}_{\mathrm{sd}}(6)=$ null, $\quad \mathrm{N}_{\mathrm{sd}}(7)=$ null, $\quad \mathrm{N}_{\mathrm{sd}}(8)=\{9\}$,

$\mathrm{N}_{\mathrm{sd}}(9)=$ null, $\quad \mathrm{N}_{\mathrm{sd}}(10)=$ null.

$\mathrm{NI}(1)=4, \quad \mathrm{NI}(2)=5, \quad \mathrm{NI}(3)=5, \quad \mathrm{NI}(4)=7, \quad \mathrm{NI}(5)=8, \quad \mathrm{NI}(6)=8, \quad \mathrm{NI}(7)=10, \quad \mathrm{NI}(8)=$ null,$\quad \mathrm{NI}(9)=$ null,

$\mathrm{NI}(10)=$ null.

Procedure for finding a non-split strong dominating set of an interval graph using an algorithm.

Step 1: $S_{1}=\{1,2,3\}$.

Step 2: $S=\{1,2,3\}$.

Step $3: \mathrm{D}_{\mathrm{st}}=\{3\}$.

Step $4: \mathrm{LI}=3$.

Step $5: \mathrm{N}_{\mathrm{sd}}(3)=\{6\}$.

Step $5.1: \mathrm{a}=\mathrm{M}\left(\mathrm{N}_{\mathrm{sd}}(3)\right)=\mathrm{M}(\{6\})=6$.

Step $5.2: \mathrm{b}=6$.

Step 5.3: $\mathrm{D}_{\mathrm{st}}=\{3\} \cup\{6\}=\{3,6\}$

Step $6: \mathrm{LI}=6$.

Step $7: \mathrm{NI}(6)=8$

Step7.1: $\mathrm{S}_{2}=\mathrm{nbd}[8]=\{7,8,9,10\}$.

Step7.2: $\mathrm{S}_{3}=\{8,9,10\}$.

Step7.3: $S_{4}=\{8,9,10\}$

Step7.4: $\mathrm{c}=9$.

Step7.5: $D_{\text {st }}=D_{\text {st }} \cup\{9\}=\{3,6\} \cup\{9\}=\{3,6,9\}$.

Step $8: V=\{1,2,3,-------10\}$ 
Step $9:\left|D_{\text {st }}\right|=3$

Step10: $\mathrm{S}_{\mathrm{N}}=\{1,2,3,4,5,6,8,10\}$

Step11 : for $\mathrm{i}=1, \mathrm{j}=2,(1,2) \in \mathrm{E}$, plot 1 to 2

for $\mathrm{i}=2, \mathrm{j}=3,(2,3) \in \mathrm{E}$, plot 2 to 3

for $\mathrm{i}=3, \mathrm{j}=4,(4,5) \in \mathrm{E}$, plot 4 to 5

$\mathrm{j}=5,(4,6) \in \mathrm{E}$, plot4 to 6

for $\mathrm{i}=4, \mathrm{j}=5,(5,6) \in \mathrm{E}$, plot 5 to 6

$\mathrm{j}=6,(5,7) \in \mathrm{E}$, plot 5 to 7

for $\mathrm{i}=5, \mathrm{j}=6,(6,7) \in \mathrm{E}$, plot 6 to 7

for $i=6, j=7,(7,8) \in E$, plot 7 to 8

for $\mathrm{i}=7, \mathrm{j}=8,(8,10) \in \mathrm{E}$, plot 8 to 10

The induced sub graph $\mathrm{G}_{1}=\left\langle\mathrm{V}-\mathrm{D}_{\mathrm{st}}\right\rangle$ is obtained.

Step12: $\mathrm{W}\left(\mathrm{G}_{1}\right)=1$

Therefore $\mathrm{D}_{\mathrm{st}}$ is the non split dominating set .

Step13: End .

Out put : $\{3,6,9\}$ is a non split strong dominating set .

Theorem 2 : If $i$ and $j$ are two intervals in $I$ such that $i \in D_{\text {st }}$ where $D_{s t}$ is a minimum dominating set of $G, j=1$ and $j$ is contained in $i$ and if there is one more interval other than $i$ that intersects $j$ then non-split strong domination occurs in G.

Proof : Let $I=\left\{\mathrm{I}_{1}, \mathrm{I}_{2}, \mathrm{I}_{3}, \mathrm{I}_{4},-----, \mathrm{I}_{\mathrm{n}}\right\}$ be an interval family. Let $\mathrm{j}=1$ be the interval contained in $\mathrm{i}$ where $\mathrm{i} \in \mathrm{D}_{\mathrm{st}}$, where $D_{\text {st }}$ is the minimum strong dominating set of $G$. Suppose $k$ is an interval, $k \neq i$ and $k$ intersect $j$. Since $i \in D_{\text {st }}$, the induced subgraph $\left\langle\mathrm{V}-\mathrm{D}_{\mathrm{st}}\right\rangle$ does not contain i. Further in $\left\langle\mathrm{V}-\mathrm{D}_{\mathrm{st}}\right\rangle$, the vertex $\mathrm{j}$ is adjacent to the vertex $\mathrm{k}$ and hence there will not be any disconnection in $\left\langle\mathrm{V}-\mathrm{D}_{\mathrm{st}}\right\rangle$. Therefore we get non split strong domination in $\mathrm{G}$.In this connection as follows an algorithm .

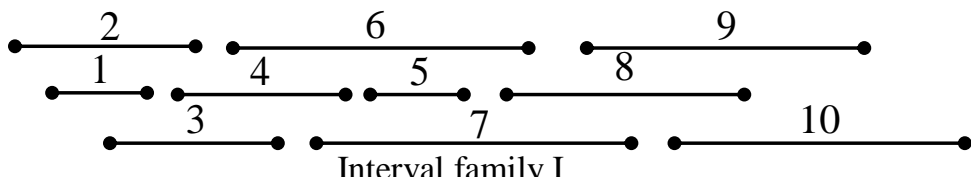

As follows an algorithm with illustration for neighbours as given interval family I. We construct an interval graph $\mathrm{G}$ from interval family $\mathrm{I}=\{1,2,3,-------10\}$ as follows

$\operatorname{nbd}[1]=\{1,2,3\}, \quad \operatorname{nbd}[2]=\{1,2,3,4\}, \quad \operatorname{nbd}[3]=\{1,2,3,4,6\}$,

$\operatorname{nbd}[4]=\{2,3,4,6,7\}, \quad \operatorname{nbd}[5]=\{5,6,7\}, \quad \operatorname{nbd}[6]=\{3,4,5,6,7,8\}$,

$\operatorname{nbd}[7]=\{4,5,6,7,8,9\}, \quad \operatorname{nbd}[8]=\{6,7,8,9,10\}, \operatorname{nbd}[9]=\{7,8,9,10\}, \operatorname{nbd}[10]=\{8,9,10\}$.

$\mathrm{N}_{\mathrm{sd}}(1)=\{2,3\}, \quad \mathrm{N}_{\mathrm{sd}}(2)=\{3,4\}, \mathrm{N}_{\mathrm{sd}}(3)=\{4\}, \quad \mathrm{N}_{\mathrm{sd}}(4)=\{7\}, \quad \mathrm{N}_{\mathrm{sd}}(5)=\{7\}, \quad \mathrm{N}_{\mathrm{sd}}(6)=\{7\}, \quad \mathrm{N}_{\mathrm{sd}}(7)=$ null, $\quad \mathrm{N}_{\mathrm{sd}}(8)=$ null $\quad \mathrm{N}_{\mathrm{sd}}(9)=$ null, $\quad \mathrm{N}_{\mathrm{sd}}(10)=$ null.

$\mathrm{NI}(1)=4, \quad \mathrm{NI}(2)=5, \quad \mathrm{NI}(3)=5, \quad \mathrm{NI}(4)=5, \quad \mathrm{NI}(5)=8, \quad \mathrm{NI}(6)=9, \quad \mathrm{NI}(7)=10, \quad \mathrm{NI}(8)=$ null,$\quad \mathrm{NI}(9)=$ null,

$\mathrm{NI}(10)=$ null.

\section{Procedure for finding a non-split strong dominating set of an interval graph using an algorithm}

Step $1: S_{1}=\{1,2,3\}$

Step $2: \mathrm{S}=\{1,2,3\}$

Step $3: \mathrm{D}_{\mathrm{st}}=3$

Step $4: \mathrm{LI}=3$

Step $5: \mathrm{N}_{\mathrm{sd}}(3)=6$

Step $6: \mathrm{a}=6$

Step $7: b=7$

Step $8: D_{\text {st }}=\{3\} \cup\{7\}=\{3,7\}$

Step $9: \mathrm{LI}=7$

Step10 $:$ NI $(7)=10$

Step10.1: $S_{2}=\{8,9,10\}$

Step10.2: $\mathrm{S}_{3}=\{10\}$

Step10.3: $\mathrm{S}_{4}=\{10\}$

Step10.4: b=10

Step10.5: $D_{\text {st }}=\{3,7,9\}$

Step11 : V=\{1,2,3,--------10 $\}$

Step12: $\left|\mathrm{D}_{\mathrm{st}}\right|=3$

Step13: $S_{N}=\{1,2,4,5,6,8,9\}$ 
Step14 : for $\mathrm{i}=1, \mathrm{j}=2,(1,2) \in \mathrm{E}$, plot 1 to 2

for $\mathrm{i}=2, \mathrm{j}=3,(2,4) \in \mathrm{E}$, plot 2 to 4

for $\mathrm{i}=3, \mathrm{j}=4,(4,5) \in \mathrm{E}$, plot 4 to 5

for $\mathrm{i}=4, \mathrm{j}=5,(5,6) \in \mathrm{E}$, plot 5 to 6

for $\mathrm{i}=5, \mathrm{j}=6, \quad(6,8) \in \mathrm{E}$, plot 6 to 8

for $\mathrm{i}=6, \mathrm{j}=7,(8,9) \in \mathrm{E}$, plot 8 to 9

The induced subgraph $\mathrm{G}_{1}=\left\langle\mathrm{V}-\mathrm{D}_{\mathrm{st}}\right\rangle$ is obtained .

Step $15: \mathrm{W}\left(\mathrm{G}_{1}\right)=1$.

Therefore $D_{\text {st }}$ is the non split strong dominating set.

Step16: End

Output : $\{3,7,10\}$ is a non split strong dominating set

Theorem 3 : Let $D_{s t}$ be a strong dominating set which is obtained by algorithm SDS. If $i, j$, $k$ are three consecutive intervals such that $\mathrm{i}<\mathrm{j}<\mathrm{k}$ and $\mathrm{j} \in \mathrm{D}_{\text {st }}$, $\mathrm{i}$ intersects $\mathrm{j}$, $\mathrm{j}$ intersect $\mathrm{k}$ and $\mathrm{i}$ interest $\mathrm{k}$ then non split strong domination occurs in $\mathrm{G}$.

Proof : Suppose $I=\left\{I_{1}, I_{2}, I_{3},-----I_{n}\right\}$ be an interval family . Let i, j, k be three consecutive intervals satisfying

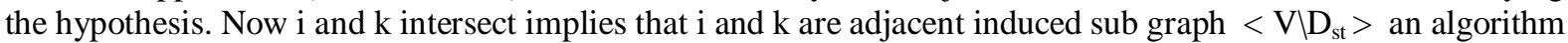
as follows

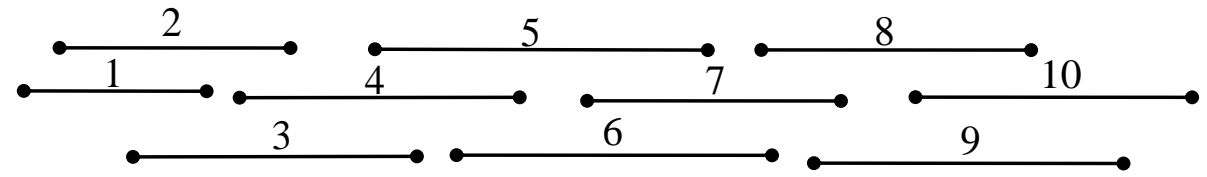

Interval family I

As follows an algorithm with illustration for neighbours as given interval family I. We construct an interval graph $\mathrm{G}$ from interval family $\mathrm{I}=\{1,2,3,-------10\}$ as follows

$\operatorname{nbd}[1]=\{1,2,3\}, \quad \operatorname{nbd}[2]=\{1,2,3,4\}, \quad \operatorname{nbd}[3]=\{1,2,3,4,5\}$,

$\operatorname{nbd}[4]=\{2,3,4,5,6\}, \quad \operatorname{nbd}[5]=\{3,4,5,6,7\}, \operatorname{nbd}[6]=\{4,5,6,7,8\}$,

$\operatorname{nbd}[7]=\{5,6,7,8,9\}, \quad \operatorname{nbd}[8]=\{6,7,8,9\}, \operatorname{nbd}[9]=\{7,8,9,10\}, \operatorname{nbd}[10]=\{9,10\}$.

$\mathrm{N}_{\mathrm{sd}}(1)=\{2,3\}, \quad \mathrm{N}_{\mathrm{sd}}(2)=\{3,4\}, \mathrm{N}_{\mathrm{sd}}(3)=$ null, $\quad \mathrm{N}_{\mathrm{sd}}(4)=$ null, $\quad \mathrm{N}_{\mathrm{sd}}(5)=$ null, $\quad \mathrm{N}_{\mathrm{sd}}(6)=$ null, $\quad \mathrm{N}_{\mathrm{sd}}(7)=$ null,

$\mathrm{N}_{\mathrm{sd}}(8)=$ null, $\quad \mathrm{N}_{\mathrm{sd}}(9)=$ null, $\quad \mathrm{N}_{\mathrm{sd}}(10)=$ null.

$\mathrm{NI}(1)=4, \quad \mathrm{NI}(2)=5, \quad \mathrm{NI}(3)=6, \quad \mathrm{NI}(4)=7, \quad \mathrm{NI}(5)=8, \quad \mathrm{NI}(6)=9, \quad \mathrm{NI}(7)=10, \quad \mathrm{NI}(8)=10, \quad \mathrm{NI}(9)=$

null, $\mathrm{NI}(10)=$ null .

Procedure for finding a non-split strong dominating set of an interval graph using an algorithm.

Step $1: S_{1}=\{1,2,3\}$

Step 2: $S=\{1,2,3\}$

Step $3: \mathrm{D}_{\mathrm{st}}=3$

Step $4: \mathrm{LI}=3$

Step $5: \mathrm{NI}(3)=6$

Step 6 : Nbd[6] $=\{4,5,6,7,8\}$

Step 6.1: $S_{3}=\{6,7,8\}$

Step $6.2: S_{3}=\{6,7,8\}$

Step 6.3: $S_{4}=\{6,7,8\}$

Step $6.4: \mathrm{c}=8$

Step 6.5: $D_{\text {st }}=\{3,8\}$

Step $7:$ LI=8

Step $8: \mathrm{NI}(8)=$ null

Step 9: V=\{1,2,3,--------10 $\}$

Step10 : $\left|D_{\text {st }}\right|=2$

Step11: $S_{N}=\{1,2,4,5,6,9,10\}$

Step12 : for $\mathrm{i}=1, \mathrm{j}=2,(1,2) \in \mathrm{E}$, plot 1 to 2 for $\mathrm{i}=2, \mathrm{j}=3,(2,4) \in \mathrm{E}$, plot 2 to 4

for $i=3, j=4,(4,5) \in E$, plot 4 to 5

$\mathrm{j}=5,(4,6) \in \mathrm{E}$, plot 4 to 6

for $\mathrm{i}=4, \mathrm{j}=5,(5,6) \in \mathrm{E}$, plot 5 to 6

$\mathrm{j}=6,(5,7) \in \mathrm{E}$, plot 5 to 7 
for $\mathrm{i}=5, \mathrm{j}=6,(6,7) \in \mathrm{E}$, plot 6 to 7

for $\mathrm{i}=6, \mathrm{j}=7,(7,9) \in \mathrm{E}$, plot 7 to 9

The induced sub graph $\mathrm{G}_{1}$ is obtained .

Step13: $\mathrm{W}\left(\mathrm{G}_{1}\right)=1$

Therefore $D_{\text {st }}$ is the non split strong dominating set.

Step14: End

Output: $\{3,8\}$ is a non split strong dominating set .

\section{Conclusions}

We studied the non-split strong domination in interval graphs. In this paper we discussed a verification method algorithm for finding a non-split strong dominating set of an interval graph.

\section{Acknowledgements}

The authors would like to express their gratitude of the anonymous referees for their suggestions and inspiring comments on this paper

\section{References}

[1]. T. W. Haynes, S.T. Hedetniemi and P.J.Slater, Fundamentals of domination in graphs, Marcel Dekker, Inc., New York (1998).

[2]. T. W. Haynes, S.T. Hedetniemi and P.J.Slater, Domination in Graphs: advanced topics, Marcel Dekker, Inc., New York (1998).

[3]. Hedetniemi, S. T, Laskar, R. C. , Bibliography on domination in graphs and some basic definitions of domination parameters, Discrete Mathematics 86 (1-3), (1990), 257-277.

[4]. Sampathkumar, E., and Pushpa Latha, Strong weak domination and domination balance in graph, Discrete Math. Vol. 161, 1996, p. 235-242.

[5]. Domke et.al, On parameters related to strong and weak domination in graphs, Discrete Math. Vol. 258, (2002) p. ,1-11.

[6]. Hattingh J.H., Henning M.A., On strong domination in Graphs, J. Combin. Math. Combin. Comput. Vol.26, (1998) p. 73-92.

[7]. Rautenbach, D., Bounds on the strong domination number, Discrete Math. Vol. 215, (2000) p. 201-212.

[8]. Kulli, V. R. and Janakiram, B., The Non-Split domination number of graph, International Journal of management and systems. Vol.19. No.2, p. 145-156, 2000.

[9]. Maheswari, B., Nagamuni Reddy, L., and A. Sudhakaraiah, Split and Non-split dominating set of circular-arc graphs, J. curr. Sci Vol 3. No.2, (2003)p. 369-372.

[10]. Dr. A. Sudhakaraiah, V. Rama Latha, E. Gnana Deepika and T.Venkateswarulu, To Find Strong Dominating Set and Split Strong Dominating Set of an Interval Graph Using an Algorithm, IJSER, Vol. 2,(2012), 1026-1034. 